\title{
Memory codes for temporal and nontemporal samples in many-to-one matching by pigeons
}

\author{
ANGELO SANTI, STEVE BRIDSON, and MARY JO DUCHARME \\ Wilfrid Laurier University, Waterloo, Ontario, Canada
}

\begin{abstract}
Pigeons were trained to match temporal ( 2 and 8 sec of keylight) and color (red and green) samples to vertical and horizontal comparison stimuli. In Experiment 1, samples that were associated with the same correct comparison stimulus displayed similar retention functions, and there was no significant choose-short effect following temporal samples. This finding was replicated in Phase 1 of Experiment 2 for birds maintained on the many-to-one mapping, and it was also obtained in birds that had been switched to a one-to-one mapping by changing the comparison stimuli following color samples. However, in Phase 2 of Experiment 2, when the one-to-one mapping was produced by changing the comparison stimuli following temporal samples, a significant chooseshort effect was observed. In Experiment 3, intratrial interference tests gave evidence of temporal summation effects when either temporal presamples or color presamples preceded temporal targets. This occurred even though these interference tests followed delay tests that failed to reveal significant choose-short effects. The absence of significant choose-short effects in Experiment 1 and in Phase 1 of Experiment 2 indicates that temporal samples are not retrospectively and analogically coded when temporal and nontemporal samples are mapped onto the same set of comparisons. The interference test results suggest that the temporal summation effect arises from nonmemorial properties of the timing system and is independent of the memory code being used.
\end{abstract}

Researchers in the area of animal cognition have developed a variety of research approaches in an attempt to determine whether the memory code for visual samples in a delayed matching-to-sample (DMTS) task is a prospective representation of the correct comparison stimulus to respond to, or a retrospective representation of sample stimulus attributes (Honig \& Thompson, 1982). These approaches include (1) comparisons of simple delayed and delayed conditional discriminations (Cohen, Galgan, \& Fuerst, 1986; Cohen, Grassi, \& Dowson, 1988; Honig \& Dodd, 1983, 1986; Honig \& Wasserman, 1981; Pontecorvo, 1985; Urcuioli \& Zentall, 1990; Wasserman, 1986; Weisman, Bruce, \& Beninger, 1987); (2) manipulating the discriminability of sample and/or comparison stimuli (Kraemer \& Roberts, 1984; Roitblat, 1980; Urcuioli \& Zentall, 1986; Zentall, Urcuioli, Jagielo, \& Jackson-Smith, 1989); (3) signalling the comparison stimulus dimension being tested (Grant \& MacDonald, 1990; Santi, Musgrave, \& Bradford, 1988; Stonebraker \& Rilling, 1984); and (4) manipulating the number and/or mapping of sample stimuli to comparisons (Grant, 1982; Santi \& Roberts, 1985; Zentall, Jagielo, Jackson-Smith, \& Urcuioli, 1987; Zentall et al., 1989). A number of researchers have acknowledged that animals

This research was supported by Grant A6378 from the Natural Sciences and Engineering Research Council of Canada to A. S. The authors would like to thank Linda Parker, Bob St. Claire-Smith, Don Wilkie, and Rob Willson for their comments at various stages in the current research. Reprints may be obtained from A. Santi, Department of Psychology, Wilfrid Laurier University, Waterlo, ON, Canada, N2L 3C5. are presumably capable of processing events either prospectively or retrospectively, depending on the demands of the task (Cohen et al., 1986; Grant \& Spetch, 1991; Honig \& Thompson, 1982; Riley, Cook, \& Lamb, 1981; Urcuioli \& Zentall, 1986).

Recently, Urcuioli, Zentall, Jackson-Smith, and Steirn (1989) have presented evidence that visual samples consisting of colors or line tilts are coded into a common, shared code by pigeons when they are associated with the same comparison stimulus. In their first experiment, the sample stimuli were color (red and green) and line orientation (vertical and horizontal) for all birds. Half the pigeons were trained with color comparisons; the other half were trained with line orientation comparisons. Comparison dimension had no effect on accuracy, but sample dimension had a small effect that did not interact with delay. Urcuioli et al. argued that associating more than one sample with the same comparison produced a common memory code for colors and line orientation samples. Strong support for this notion was obtained in subsequent transfer tests. Pigeons initially trained to match color and line orientation samples to vertical and horizontal comparison stimuli learned new comparison associations to the color samples. When the pigeons were tested on their ability to match the line orientation samples to the new comparisons, immediate transfer was obtained. Positive transfer occurred when sample-comparison associations during testing were consistent with the hypothesized common code established earlier, and negative transfer occurred when the associations tested were inconsistent with the common code. These results provide converging evi- 
dence with those reported by Grant (1982), Santi and Roberts (1985), and Zentall et al. (1989)-that some form of common coding is promoted by a many-to-one (MTO) mapping of samples to comparison stimuli in DMTS.

Research on memory for temporal events in pigeons has also been concerned with the nature of the memory code. A phenomenon known as the choose-short effect arises in delayed matching when variable-length delay intervals are presented following presentation of temporal samples. As the delay interval increases, pigeons show an increasing tendency to peck the comparison stimulus corresponding to the short-duration sample (Spetch, 1987; Spetch \& Rusak, 1989; Spetch \& Wilkie, 1982). The subjective shortening model, first proposed by Spetch and Wilkie (1983), maintains that pigeons retain temporal information in a retrospective and analogical fashion. This model accounts for the choose-short effect by assuming that the subjective duration of the sample decreases during the delay interval. In opposition to this view, some have argued that temporal information is categorically coded along a nontemporal dimension (Kraemer, Mazmanian, \& Roberts, 1985; Parker \& Glover, 1987). This could be a prospective categorical code (e.g., peck red or peck green) or a retrospective categorical code (e.g., short or long).

Recently, strong evidence has been found that pigeons remember event durations in an analogical and retrospective fashion. Spetch and Sinha (1989) used an intratrial proactive interference design to distinguish between an analogical code of event duration and a categorical code (either prospective or retrospective). Short (2-sec) or long $(10-\mathrm{sec})$ target samples were preceded on test trials by a short or long presample. The durations were consistent on some trials (S-S or L-L) and inconsistent on others (S-L, L-S). The categorical model predicts enhanced accuracy on S-S trials and an interference effect on S-L trials. The analogical model predicts reduced accuracy on S-S trials and enhanced accuracy on S-L trials because the presample and target sample durations summate in working memory. The results were consistent with the predictions of the analogical model.

Further evidence for the analogical coding of event durations was provided by Wilkie and Willson (1990). They required pigeons to discriminate $2-, 8-$, and $10-$ sec presentations of light. Choices of red, orange, and green keys were correct after 2, 8, and $10 \mathrm{sec}$, respectively. Differences in discriminability between the samples and comparison stimuli permitted Wilkie and Willson to examine discriminal distance scores. As task difficulty increased, the change in the discriminal distance scores was consistent with retrospective coding of event durations, not prospective coding. Wilkie and Willson also adapted Spetch and Sinha's (1989) proactive interference design to their discriminal distance study and obtained results consistent with the hypothesis that the retrospective code was analogical, not categorical.

While pigeons appear to code temporal information in a retrospective and analogical fashion when a choice
DMTS task is used (Spetch \& Sinha, 1989; Wilkie \& Willson, 1990), this does not appear to be the case when a successive DMTS task is used. Grant and Spetch (1991) have shown that pigeons trained and tested in a successive task do not show a tendency to respond to test stimuli at long delays as if the sample had been short. That is, it does not appear that remembered duration controls responding to the test stimuli in the successive task. Grant and Spetch suggested that in this task temporal samples are prospectively coded in terms of instructions to respond and/or not respond to particular test stimuli.

Recently, Ducharme and Santi (1993) have shown that pigeons do not code temporal samples retrospectively and analogically when they are differentially associated with food and no-food outcomes in a choice MTS task. Instead, event durations are remembered in terms of the corresponding outcome expectancies.

The purpose of the present research was to determine whether an MTO procedure with the choice task would also result in pigeons coding event durations in a nonanalogical fashion. This procedure was used because Urcuioli et al. (1989) have shown that pigeons do not code visual samples in a unique, nonoverlapping fashion when the samples are associated with the same comparison stimulus. Rather, the pigeons develop a shared, common code for events associated with the same comparisons. In Experiment 1 , retention functions for temporal and color samples mapped onto the same comparisons were compared. In Experiment 2, the birds were shifted to a oneto-one (OTO) mapping condition by changing the comparison stimuli for either the temporal dimension or the nontemporal dimension, and retention functions were obtained. In Experiment 3, intratrial proactive interference tests were conducted.

\section{EXPERIMENT 1}

With an MTO procedure, pigeons were trained to match temporal ( 2 and $8 \mathrm{sec}$ of keylight) and color (red and green) samples to line tilt comparisons (vertical and horizontal lines). After training, the rates of forgetting for both temporal and color samples were assessed. If pigeons formed a common code for temporal and color samples, the retention functions for both sample dimensions should be similar. In addition, for temporal samples, there should be no evidence of a choose-short effect even at long delays.

\section{Method}

\section{Subjects}

Sixteen experimentally naive male White Carneaux pigeons, maintained at approximately $80 \% \pm 20 \mathrm{~g}$ of their ad-lib weights and housed individually with constant access to grit and water, served as subjects.

\section{Apparatus}

Four Coulbourn modular operant test cages (Model E10-10), housed individually in isolation cubicles (Model E10-20), were used. The cubicles were equipped with a ventilation fan and baffled air intake exhaust systems. Each test cage was equipped with three 
horizontally aligned, clear plastic keys, behind which projectors could display stimuli (red or green field, a white vertical, a white horizontal, or white crossed diagonal lines on a black background, or a black dot on a white background) onto a frosted rear projection screen (Coulbourn Model E21-18). Directly below the center key was a $5.7 \times 5 \mathrm{~cm}$ opening that provided access to a hopper filled with mixed grain (Coulbourn Model E14-10). A houselight was located $6.5 \mathrm{~cm}$ above the center key and installed so that the light was directed upward to reflect from the top of the cage (Coulbourn Model E11-01 with Bulb SL1819X). All experimental events and response measures were controlled by a microcomputer located in an adjacent room.

\section{Procedure}

The experimental procedure was divided into three phases: preliminary training, acquisition of 0-sec-delay MTS, and DMTS testing.

Preliminary training. All pigeons were trained to eat mixed grain from an illuminated food hopper. Following hopper training, the pigeons were autoshaped to peck a white vertical line, a white horizontal line, or a black dot on a white background. The black dot was always presented on the center key; the two line tilts were presented on the right or left side keys. The order of stimulus presentation was randomized individually for each bird. Each stimulus remained on until it was pecked or $8 \mathrm{sec}$ had elapsed, whichever occurred first. The reinforcer consisted of access to a hopper filled with mixed grain. The duration of food access was gradually reduced from 8 to $3 \mathrm{sec}$ during autoshaping. A 25 -sec intertrial interval (ITI) followed each reinforcement. The houselight remained off throughout the experiment. Each of the seven sessions of autoshaping consisted of 120 trials. Three birds required some manual shaping in order to initiate keypecking.

Acquisition of 0-sec delay matching. Acquisition of MTO matching with temporal and color samples immediately followed preliminary training. All pigeons were trained with the same set of sample stimuli (red or green colored fields, and 2- or 8-sec illumination of crossed diagonal lines) and the same set of comparison stimuli (vertical and horizontal lines) at a 0-sec delay. Each trial began with the warning stimulus (black dot on a white background) presented on the center key. A single peck to it resulted in presentation of one of the four sample stimuli on the center key. The color samples were presented for $4 \mathrm{sec}$, because this is an approximate estimate of the point of subjective equality between 2- and 8-sec temporal samples for pigeons (Spetch \& Wilkie, 1983). Immediately following the offset of the sample stimulus, the comparison stimuli were presented. For 8 birds, a peck to the vertical line comparison following either a red or a 2-sec sample and a peck to the horizontal line comparison following a green or an 8-sec sample was correct. For the remaining 8 birds, the relationship between the samples and the correct comparison stimulus was reversed. A single peck to the comparison stimuli turned them off and if correct permitted a 2 to 3-sec access to mixed grain. Reinforcement time was varied slightly between birds in order to maintain them at their $80 \%$ adlib weight. When necessary, supplementary feedings of Purina Pigeon Chow occurred after the experimental sessions. Incorrect responses to the comparison stimuli added $3 \mathrm{sec}$ to the 20-sec ITI.

Within each block of eight trials, all combinations of the four sample stimuli with vertical and horizontal line comparisons on the left and right side keys occurred once. The order of presentation was randomized individually for each bird. All birds received 144 trials per session. At the end of 30 sessions of MTO training with temporal and color samples, all birds were matching at $85 \%$ or better on both dimensions.

DMTS testing. DMTS testing followed acquisition of the 0-secdelay task. Delays of $0,5,10$, and $15 \mathrm{sec}$ were scheduled across the 144 trials in each of the 15 test sessions. Within each block of 72 trials, 12 trials for each sample occurred at the 0 -sec delay and 2 trials for each sample occurred at each of the other delays
$(5,10$, and $15 \mathrm{sec})$. This distribution of delays was used so that the reference memory of the sample durations and their associations with the comparison stimuli established during 0 -sec delay training would remain relatively stable during testing (see Spetch $\&$ Wilkie, 1983). All other parameters remained the same as those described for acquisition.

\section{Results}

The pigeons required significantly fewer sessions to match at $85 \%$ or greater accuracy with color samples than with temporal samples $[F(1,15)=21.49, p<.0001]$. It took an average of 11.6 sessions with color samples and 17.8 sessions with temporal samples. The mean percentage of correct matching responses obtained during acquisition was collapsed into blocks of three sessions and are shown in Figure 1. The data indicated that the matching of color samples was acquired faster than the matching of temporal samples. A 2 (sample dimension) $\times 10$ (blocks of 3 sessions) analysis of variance (ANOVA) performed on these data revealed a significant effect of sample dimension $[F(1,15)=39.33, p<.0001]$, blocks $[F(9,135)=196.32, p<.0001]$, and a sample dimension $\times$ block interaction $[F(9,135)=11.15, p<.0001]$. Simple main effects analysis showed that while there was no difference in accuracy for color and temporal samples in Block 1 of acquisition, on all remaining blocks color samples were matched more accurately than temporal samples. In the block of sessions that preceded delay testing, accuracy was $97.5 \%$ for color samples and $94.5 \%$ for temporal samples.

Figure 2 shows the delay testing data for the two temporal samples (short and long) and the two color samples (red and green). Accuracy declined markedly between the 0 - and 5-sec delays, and at delays greater than $0 \mathrm{sec}$ it was higher when the sample was red or short than when the sample was green or long. An ANOVA performed on these data included sample dimension (color or temporal), delay interval, and comparison mapping as factors. The comparison mapping variable reflected whether a particular comparison stimulus was correct after both red and short samples or after both green and long samples. For each bird, red and short samples were mapped onto one choice alternative (either vertical or horizontal line) and green and long samples were mapped onto the other choice alternative. The analysis revealed significant effects of delay $[F(3,45)=831.45, p<.0001]$, sample dimension $[F(1,15)=6.53, p<.05]$, and a sample dimension $\times$ comparison mapping interaction $[F(1,15)=5.32, p<$ $.05]$. Simple main effects analysis indicated that matching accuracy was significantly higher for red samples than for short samples $[F(1,15)=9.77, p<.05]$ and for long samples $[F(1,15)=4.58, p<.05]$, and marginally higher than for green samples $[F(1,15)=3.98, p<$ $.065]$. There was no significant difference in accuracy between green samples and long samples $[F(1,15)<1]$. Most importantly, there was no significant difference in accuracy between short- and long-sample trials $[F(1,15)=$ 1.85]. None of the interactions involving delay were statistically significant. Examination of Figure 2 might lead 


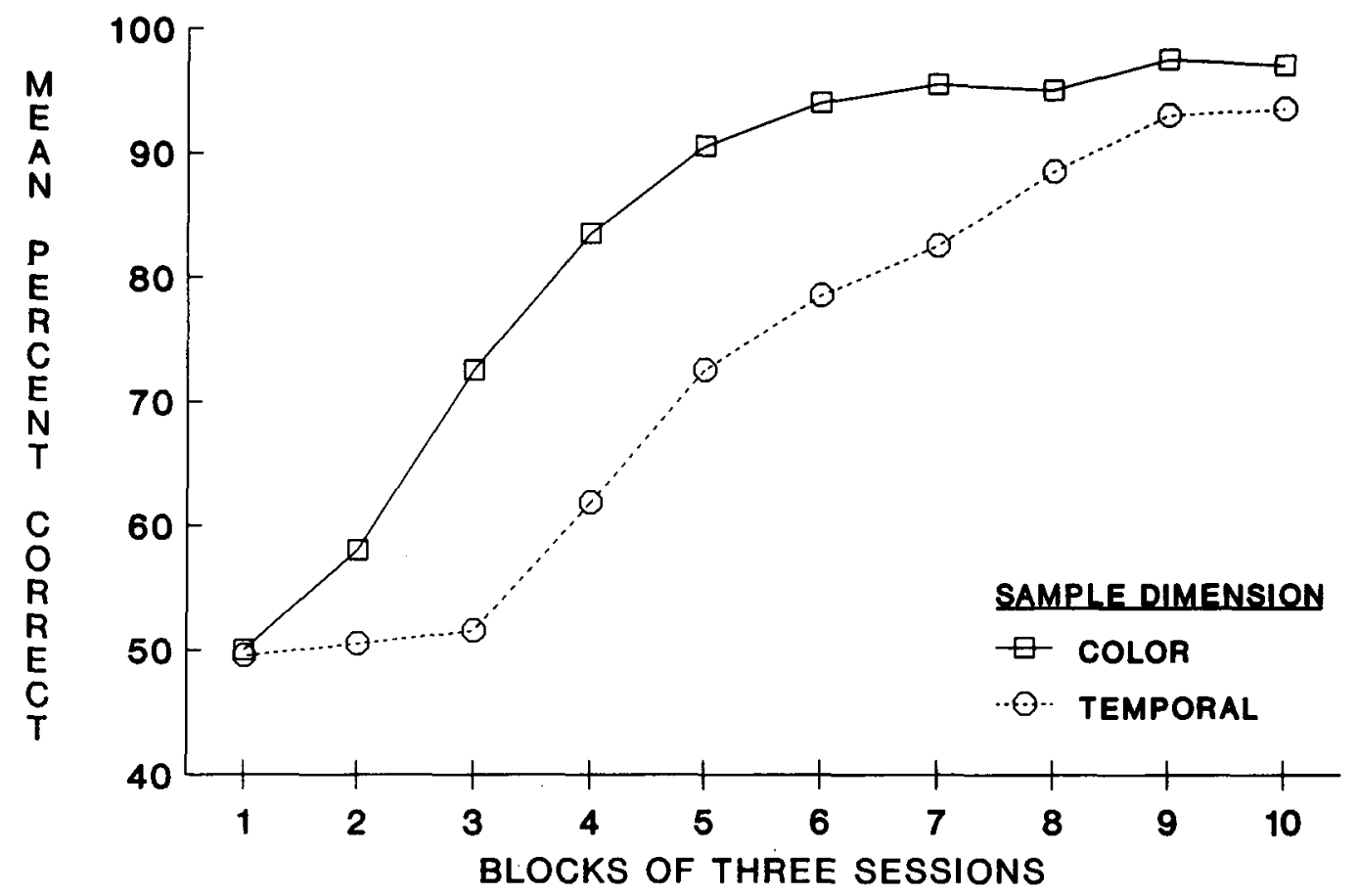

Figure 1. Mean percentage of correct responses for color and temporal samples during acquisition of many-to-one matching-to-sample in Experiment 1, averaged over blocks of three sessions.

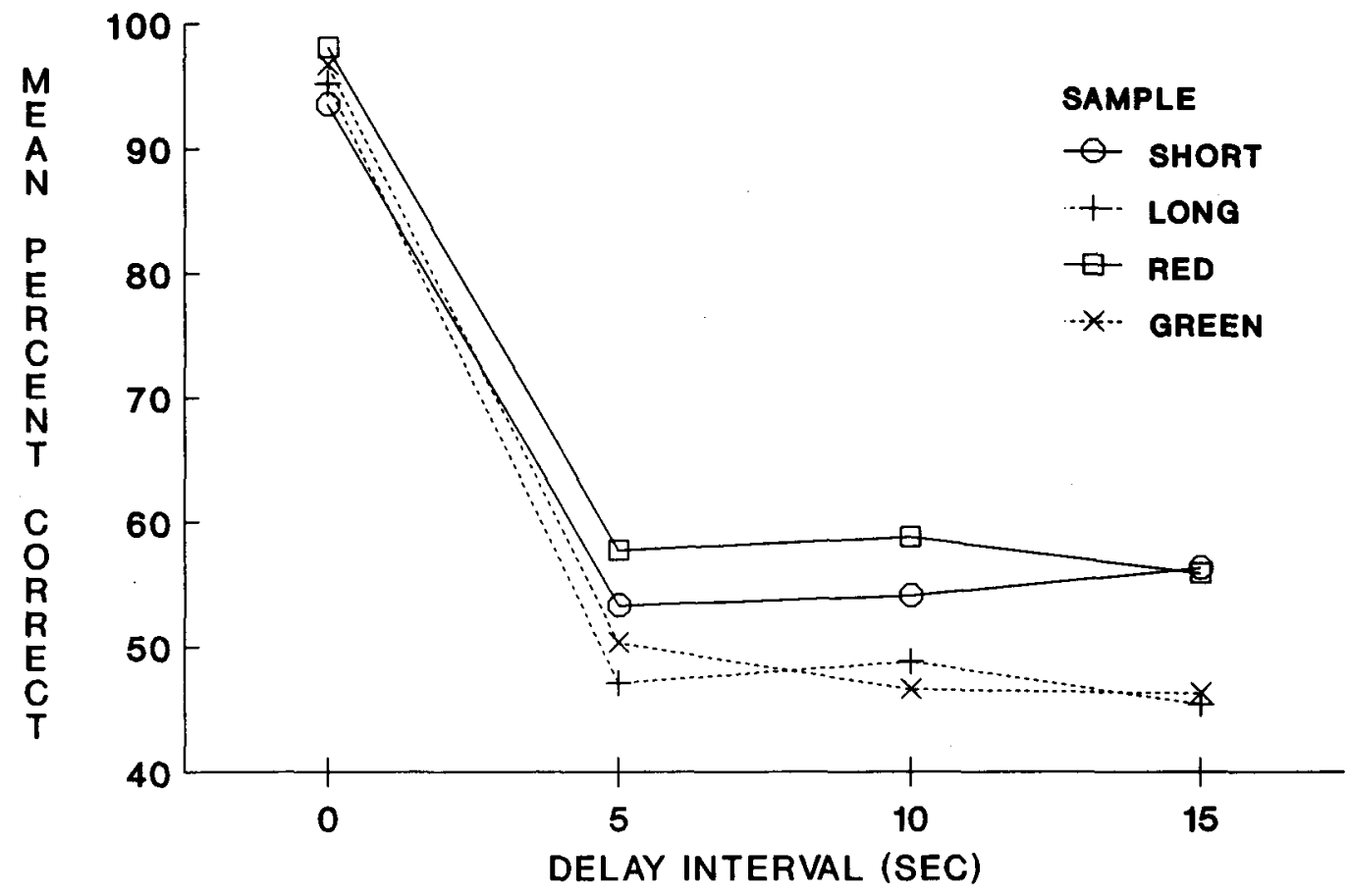

Figure 2. Mean percentage of correct responses for short, long, red, and green samples during delay interval testing in Experiment 1. 
one to expect a significant main effect of comparison mapping, because its effect relative to that associated with sample dimension seems large. However, considerably more error variance was associated with comparison mapping than with sample dimension. Additional analyses indicated that of the 16 birds, 14 displayed similar choice alternative biases. Ten birds showed higher accuracy for the comparison mapped to red and short samples, while 4 showed higher accuracy for the comparison mapped to green and long samples.

\section{Discussion}

The main purpose of Experiment 1 was to determine whether an MTO mapping of temporal and color samples onto the same set of comparisons would affect the way in which event durations are coded in memory. If pigeons coded the sample stimuli retrospectively, as the delay interval was increased, we would expect a bias to choose short following temporal samples, but no systematic bias to a particular comparison following color samples. The pattern of results was not consistent with this prediction. It is unlikely that the failure to obtain a significant chooseshort effect was due to a floor effect at delay intervals greater than zero. The delays used in the present experiment were similar to those which in other studies have consistently resulted in significant choose-short effects (Ducharme \& Santi, 1993; Grant \& Spetch, 1991; Spetch, 1987; Spetch \& Rusak, 1989; Spetch \& Wilkie, 1983). In other studies, with similar delay intervals, accuracy on short-sample trials has been much higher than it was in the present experiment. Furthermore, at long delay intervals, in other studies, there has been a tendency for accuracy on long-sample trials to fall significantly below chance, so it may be inappropriate to view $50 \%$ correct matching as a floor for long-sample trials. For these reasons, it is unlikely that the absence of a choose-short effect was due to a floor effect. Instead, the absence of a significant choose-short effect and the similarity in the retention functions for temporal and visual samples mapped onto the same comparison stimulus suggests that temporal samples were not retrospectively and analogically coded when an MTO mapping was used. Given the previous findings that some form of common coding is promoted by an MTO mapping of samples to comparison stimuli in DMTS (Grant, 1982; Santi \& Roberts, 1985; Urcuioli et al., 1989; Zentall et al., 1989), it is highly likely that pigeons formed a common code for temporal and visual samples in the present experiment. The finding that matching accuracy was significantly higher with red samples than with green samples or either temporal sample could be due to red's being more discriminable than the other samples and capable of eliciting the correct common code faster or with a higher probability.

The acquisition results showed higher matching accuracy for color samples than for temporal samples. Bowers and Richards (1990) obtained a similar result in an experiment in which pigeons were presented with 5or $30-\mathrm{sec}$ durations of a red or green sample. For one group of birds, the color of the sample was the relevant cue; for the other group, the duration of the sample was the relevant cue. Bowers and Richards found that acquisition was more rapid for the color-relevant group than for the duration-relevant group, and this difference in accuracy was maintained across various delays in subsequent test sessions. In contrast, the present data indicated that the difference in accuracy for color and temporal samples disappeared at longer delay intervals.

The difference between the retention functions for temporal samples in the present experiment and those obtained in previous experiments with an OTO choice procedure suggests that the nature of the mapping procedure was the important variable that produced these differences. Additional evidence for the importance of this variable was obtained in Experiment 2.

\section{EXPERIMENT 2}

Experiment 2 was undertaken to determine how important the MTO mapping was for the results obtained in the previous experiment. In Phase 1 , half the birds were shifted to an OTO mapping condition in which temporal samples continued to be mapped onto line comparisons and the comparisons for color samples were shifted to colors. The other half of the birds were maintained on the MTO mapping used in Experiment 1. Delay testing was then carried out to assess the effect of the OTO mapping for the OTO birds and to assess the reliability of the previous delay data for the MTO birds. In Phase 2, the remaining MTO birds were shifted to an OTO mapping condition in which color samples continued to be mapped onto line comparisons and temporal samples were mapped onto color comparisons. This permitted a comparison of two different ways in which the OTO condition could be produced, by changing the mapping for color samples (Phase 1) or changing the mapping for the temporal samples (Phase 2). It is possible that producing the OTO condition by changing the comparison stimuli for temporal samples would be more likely to alter the coding of temporal samples, because the birds would have to acquire new sample-comparison associations. If this results in the birds' reverting to a retrospective and analogical coding of temporal samples, then a choose-short effect should be obtained during delay testing. Indirect evidence that changing the comparison stimuli for temporal samples would be particularly effective comes from a study by Grant (1991), in which the coding of food and no-food samples was investigated. With these samples, pigeons demonstrated more rapid forgetting on food trials than on no-food trials. This appears to be due to asymmetrical coding of food samples with responding to the comparison associated with the no-food sample occurring by default. Grant showed that equivalent rates of forgetting on food and no-food trials could be obtained by training birds in an MTO procedure with samples of food, no food, horizontal line, and vertical line mapped onto red and green comparison stimuli. Differential rates of forgetting 
were subsequently obtained in these birds when an OTO procedure was introduced by changing the comparison stimuli associated with food and no-food samples. This suggests that a choose-short effect for temporal samples should be obtained in our study if the switch from the MTO to OTO procedure is accomplished by changing the comparisons for temporal samples.

\section{Method}

\section{Subjects and Apparatus}

The subjects and the apparatus were the same as in Experiment 1. Prior to participating in Experiment 2, the birds had participated in a transfer of training experiment in which an MTO mapping was used, but the results of that study were inconclusive and are not being reported.

\section{Procedure}

Phase 1. All birds received nine sessions ( 72 trials each) of MTO matching with temporal and color samples mapped onto line orientation comparisons as described for the sessions prior to delay testing in Experiment 1. There was no correction procedure and the delay interval was $0 \mathrm{sec}$. At the end of these nine sessions, accuracy was $97.3 \%$ for the color samples and $93.8 \%$ for the temporal samples. Eight of the birds were then shifted to an OTO condition. All aspects of the OTO procedure were the same as for the MTO procedure, except that color samples were now mapped onto color comparisons. Pecking the red comparison was the correct response following a red sample; pecking green was correct following a green sample. The MTO birds continued to receive training as previously described. This phase of training continued for 25 sessions.

Delay testing. Retention was evaluated at delay intervals of 0 , 1,3 , and $9 \mathrm{sec}$. Sixteen test sessions of 72 trials each were given. Within each session, 12 trials for each sample occurred at the 0 -sec delay and 2 trials for each sample occurred at each of the other delays $(1,3$, and $9 \mathrm{sec})$. All other parameters remained the same as those described for training in Phase 1 .

Phase 2. Following these delay tests, all birds were given a series of interference test sessions, but in order to facilitate exposition these will be reported as Experiment 3 . After the interference tests, only the 8 birds that had always been trained with an MTO mapping continued in the study. They received an additional 10 sessions (72 trials each) of MTO matching with temporal and color samples mapped onto line orientation comparisons as described previously. These birds were then shifted to an OTO mapping in which all aspects of the procedure were the same as for the MTO procedure, except that temporal samples were now mapped onto color comparisons. Pecking the red comparison was the correct response following a short sample; pecking green was correct following a long sample. This phase of training continued for 20 sessions.

Delay testing. Retention was evaluated at delay intervals of 0 , 1, 3, and 9 sec. Eighteen test sessions of 72 trials each were given. Within each session, 12 trials for each sample occurred at the 0 -sec delay and 2 trials for each sample occurred at each of the other delays $(1,3$, and $9 \mathrm{sec})$. All other parameters remained the same as those described for training in Phase 1.

\section{Results}

\section{MTO/OTO Training Accuracy}

Figure 3 shows accuracy on the session prior to and after the session of the shift to color comparisons following color samples for the OTO group in Phase 1 and following temporal samples for the OTO group in Phase 2. Subjects in both of the OTO groups showed a drop in accuracy on the shifted sample dimension to chance levels

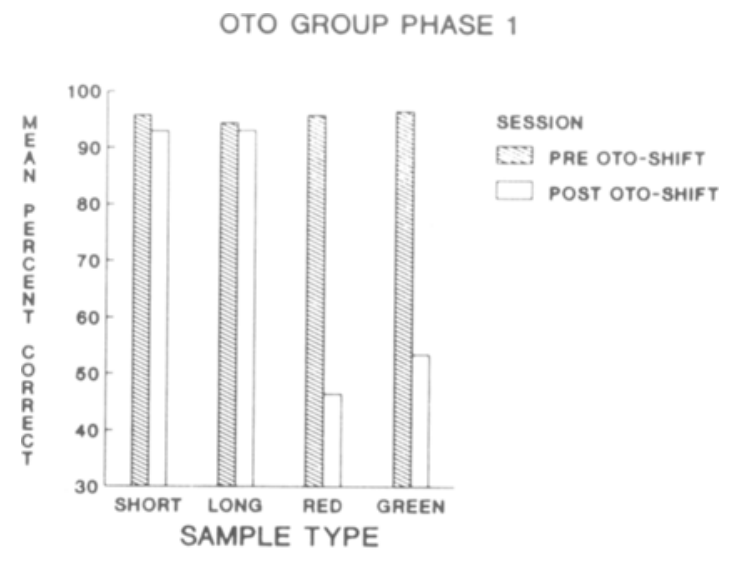

OTO GROUP PHASE 2

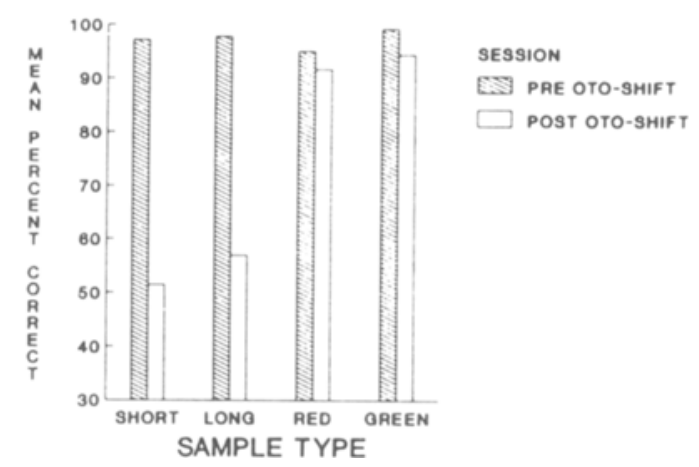

Figure 3. Mean percentage of correct responses for short, long, red, and green samples on the session before and after the introduction of color comparison stimuli for color samples (OTO Group: Phase 1) and for temporal samples (OTO Group: Phase 2).

when color comparisons were presented following color samples in Phase 1 or temporal samples in Phase 2. The disruption in accuracy on the shifted sample dimension did not affect accuracy on the nonshifted sample dimension for either OTO group. The OTO group in Phase 1 maintained high levels of accuracy on both short- and long-sample trials; the OTO group in Phase 2 maintained high levels of accuracy on both red and green sample trials.

\section{Delay Testing}

Figure 4 shows the delay testing data for the two temporal samples (short and long) and the two color samples (red and green). The data from the Phase 1 delay tests are shown in the upper half of the figure and the data from the Phase 2 delay test are shown in the bottom half. Accuracy declined less abruptly with these delay intervals than it did in Experiment 1 with the longer delays. During the Phase 1 delay testing, neither the MTO nor the OTO group displayed a choose-short effect. However, during Phase 2 , the OTO group created by changing the comparisons for temporal samples did display a significant choose-short effect.

An ANOVA performed independently on the data for the MTO group and the OTO group in Phase 1 revealed 
MTO GROUP: PHASE 1

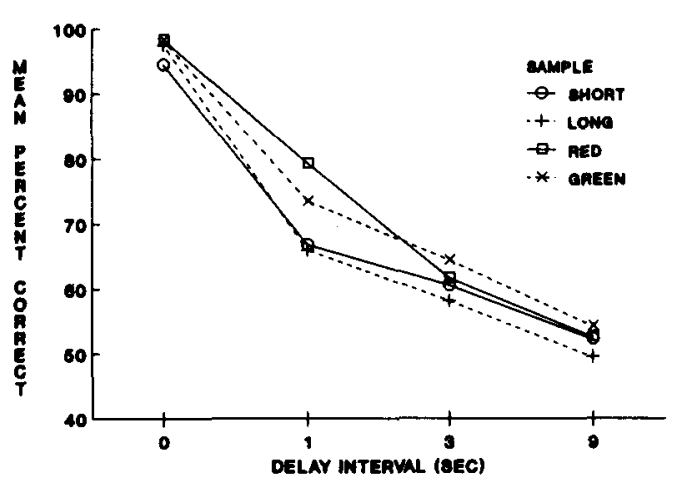

OTO GROUP: PHASE 1

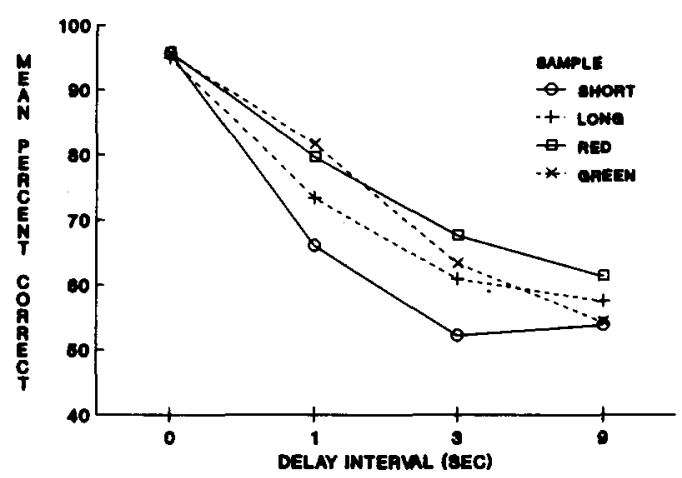

OTO GROUP: PHASE 2

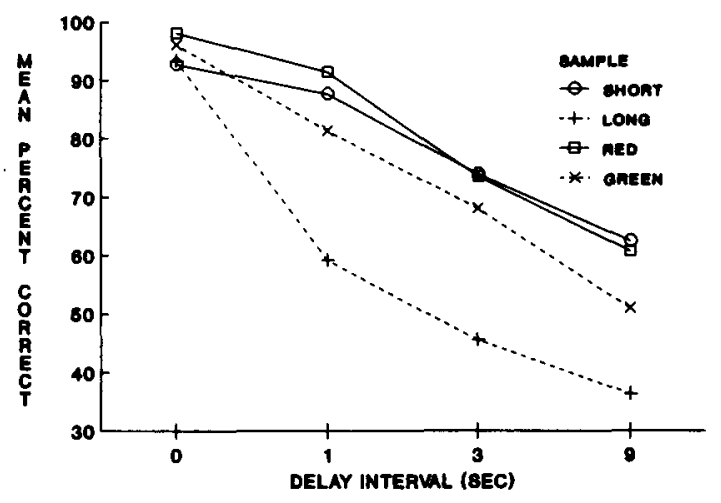

Figure 4. Mean percentage of correct responses for short, long, red, and green samples during delay interval testing in Phase 1 (top) and Phase 2 (bottom) of Experiment 2.

significant effects of delay for both groups $[F(3,21)=$ 112.14 and $F(3,21)=83.29$, respectively, $p<.0001$ ] However, in both groups, there was no significant difference in accuracy on short- and long-sample trials $(F \mathrm{~s}<$ 1 ), and no interaction of sample type $\times$ delay on the temporal dimension $(F \mathrm{~s}<1)$. However, for the OTO group in Phase 2, in addition to a significant effect of delay $[F(3,21)=81.21, p<.0001]$, there was higher accuracy on short-sample trials than on long-sample trials $[F(1,7)=$ $39.88, p<.01]$, and a significant interaction of sample type $\times$ delay on the temporal dimension $[F(3,21)=5.47$, $p<.01]$. Analysis of simple main effects revealed that a significant choose-short bias occurred at the 1-, 3-, and 9-sec delay intervals $[F(1,7)=14.58, F(1,7)=32.86$, and $F(1,7)=10.38$, respectively], but not at the 0 -sec delay interval $(F<1)$.

\section{Discussion}

A significant choose-short effect was not observed during delay testing for the MTO group or the OTO group in Phase 1. For the MTO group, this replicates the findings from Experiment 1 and suggests that temporal samples are not analogically coded when an MTO mapping is used. These findings are consistent with the idea that the temporal and nontemporal samples were commonly coded. In addition, it is clear from the data of the OTO group in Phase 1 that changing the comparison mapping for nontemporal samples did not result in the temporal samples' being analogically coded. There was no evidence of a choose-short effect in the data of the OTO group in Phase 1 , even though temporal and nontemporal samples no longer shared the same comparisons. This suggests that the coding of temporal samples in memory remained constant as long as the comparison stimuli for these samples were not changed.

In Phase 2, producing the OTO condition by changing the comparison stimuli for the temporal samples themselves resulted in a choose-short effect during delay testing. This is generally regarded as indicative of event durations' being analogically coded in memory. These findings parallel those reported by Grant (1991). He showed that equivalent rates of forgetting on food and nofood trials could be obtained by training birds in an MTO procedure with samples of food, no food, horizontal line, and vertical line mapped onto red and green comparison stimuli. Differential rates of forgetting were subsequently obtained in these birds when an OTO procedure was introduced by changing the comparison stimuli associated with food and no-food samples. Differential rates of forgetting for short and long sample durations was obtained 
in our experiment when the switch from the MTO to the OTO procedure was accomplished by changing the comparisons for temporal samples.

\section{EXPERIMENT 3}

Intratrial proactive interference tests have been used by Spetch and Sinha (1989) and Wilkie and Willson (1990) to distinguish between analogical coding and categorical coding of temporal samples. Short $(2 \mathrm{sec})$ or long $(8 \mathrm{sec})$ target samples were preceded on test trials by a short $(2 \mathrm{sec})$ or long $(8 \mathrm{sec})$ presample. The durations were consistent on some trials (S-S or L-L) and inconsistent on others (S-L, L-S). These studies indicated that temporal samples were coded analogically, because the reduced accuracy observed on S-S trials and the enhanced accuracy observed on S-L trials were consistent with the idea that the presample and target sample durations summated in working memory. However, Kraemer and Roper (1992) trained pigeons on a temporal MTS task in which each of four combinations of signal type (red or white light) and duration $(2$ or $10 \mathrm{sec}$ ) was mapped onto a different choice stimulus. Interference tests were then conducted in which the presample and target sample durations were either the same (red-red or white-white) or different signal types (red-white or white-red). Unlike Spetch and Sinha (1989), who found a summation effect with both same-signal and different-signal compounds, Kraemer and Roper (1992) found that pigeons tended to summate the two durations when the same signal was presented twice, but not when two different signals appeared. In Spetch and Sinha's study, pigeons were trained with an MTO mapping procedure in which the same duration of two different signal types was mapped onto the same comparison stimulus, whereas in Kraemer and Roper, unique comparison sets were associated with different signal types. Thus, the presence versus absence of a mapping arrangement that encourages common coding appears to determine whether or not summation effects are observed when different signal types are presented.

Because the MTO mapping condition used in Experiment 1 and in Phase 1 of Experiment 2 resulted in the temporal samples' being coded nonanalogically, we thought that it would be interesting to examine proactive interference effects. On the basis of the original rationale provided for these tests by Spetch and Sinha (1989), we anticipated that we would not obtain summation effects. Instead, there should be enhanced accuracy on S-S trials and interference effects on S- $L$ trials, because temporal samples were presumably being commonly coded in our MTO procedure. In addition to interference tests that consisted of temporal presamples and temporal target samples, interference tests were also conducted with color presamples and temporal targets, as well as temporal presamples and color targets.

\section{Method}

\section{Subjects and Apparatus}

The subjects and the apparatus were the same as in the previous experiments.

\section{Procedure: Interference Tests}

Following the delay testing conducted in Phase 1 of Experiment 2, all 16 birds were given 12 sessions of training with a 0 -sec delay interval in order to reestablish high levels of matching accuracy. Interference tests on trials involving temporal presamples and temporal target samples were then administered for 20 sessions. Each session consisted of 80 trials. Within each block of 5 trials, 4 trials were the same as baseline training conditions $(0-\sec$ delay and no presample), and 1 trial was an interference trial. Consequently, there were 64 baseline trials and 16 interference trials within each session. The interference trials consisted of a temporal presample and a temporal target sample separated by an interstimulus interval of $2 \mathrm{sec}$ and a delay interval of $0 \mathrm{sec}$. A correction procedure was not used. The 16 interference trials consisted of equal numbers of the four presample-target sample combinations: S-S, L-L, L-S, and S-L.

After the interference tests with temporal presample-temporal target samples were completed, all birds were returned to baseline training conditions ( 0 -sec delay and no presample) for at least 26 sessions. Interference tests were then initiated in which test sessions involving temporal presamples-color target samples alternated with test sessions involving color presamples-temporal target samples. A total of 20 interference test sessions were administered (10 temporal-color, 10 color-temporal). All aspects of the interference test sessions were similar to those described above for the temporal presample-temporal target tests.

\section{Results}

Figure 5 summarizes the results from the interference test sessions. The results of tests with temporal presamples and temporal target samples are presented in the upper half of Figure 5, while the results of tests with color presamples and temporal target samples and those with temporal presamples and color target samples are presented in the lower left and lower right of Figure 5, respectively. For ease of presentation, all of the interference test data has been averaged across all 16 subjects. This was undertaken because all of the interference tests were conducted immediately after Phase 1 of Experiment 2 and both the MTO and OTO group appeared to code temporal samples nonanalogically in that phase. In addition, an analysis of the results of interference testing revealed equivalent effects in both groups. Relative to performance on trials with no presample, accuracy on test trials with short target samples was decreased by short or long presamples, whereas accuracy on test trials with long target samples was not affected by short or long presamples. An ANOVA showed a significant effect of presample duration $[F(2,28)=65.11, p<.0001]$ and target sample duration $[F(1,14)=69.12, p<.0001]$, and a significant presample duration $\times$ target sample duration interaction $[F(2,28)=87.25, p<.0001]$. The group factor was not significant, and it did not interact with any other factor. Simple main effect analysis of the 


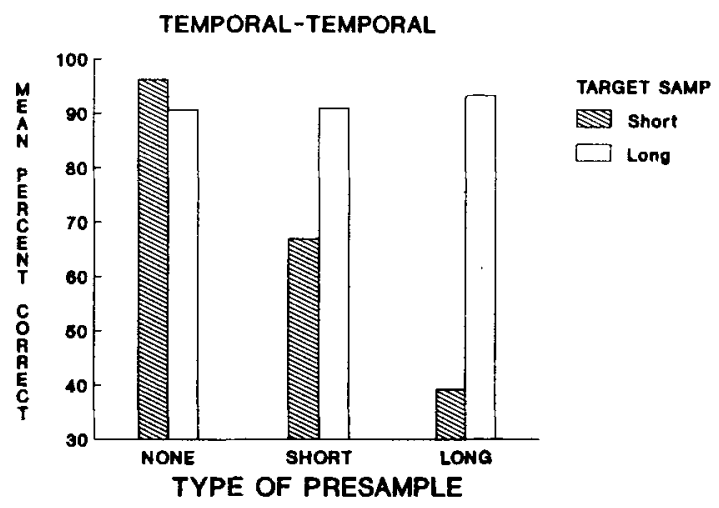

COLOR-TEMPORAL

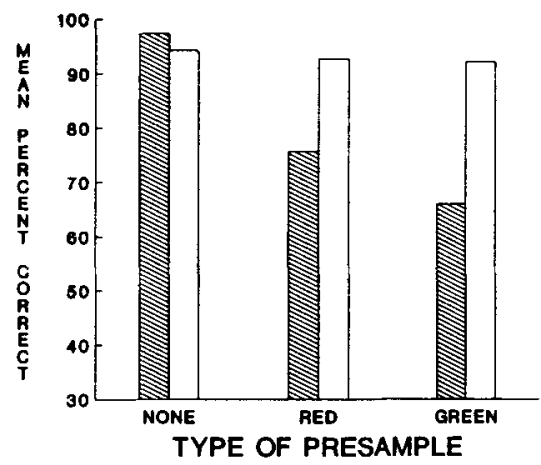

TEMPORAL-COLOR

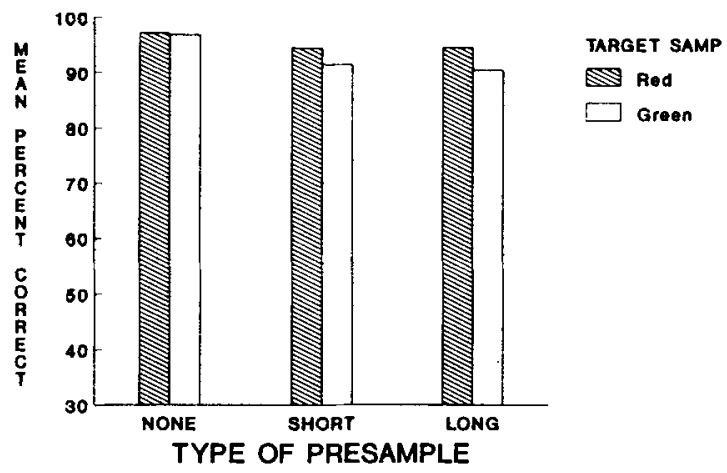

Figure 5. Mean percentage of correct responses for target samples as a function of the type of presample during interference tests in Experiment 3. Temporal presamples-temporal target sample data are shown in the upper half of the figure. Color presamples-temporal target sample data are shown in the lower left, and temporal presamples-color target sample data are shown in the lower right.

presample $\times$ target sample interaction indicated that presample duration had a significant effect on accuracy when the target sample was short $[F(2,28)=85.21, p<$ $.0001]$, but not when it was long $[F(2,28)=1.76]$.

As can be seen in the lower left graph of Figure 5, color presamples had an effect similar to that of temporal presamples on the accuracy with which temporal target samples were matched. Relative to accuracy on trials with no presample, accuracy on test trials with short target samples was decreased by red or green presamples, whereas accuracy on test trials with long target samples was not affected by red or green presamples. An ANOVA showed a significant effect of type of presample $[F(2,28)=$ $17.83, p<.0001]$ and target sample duration $[F(1,14)=$ $20.71, p<.001]$, and a significant type of presample $\times$ target sample duration interaction $[F(2,28)=21.21, p<$ $.0001]$. The group factor was not significant and it did not interact with any other factor. Simple main effect anal$y$ sis of the presample $\times$ target sample interaction indicated that type of presample had a significant effect on accuracy when the target sample was short $[F(2,28)=$ $22.39, p<.0001]$, but not when it was long $[F(2,28)<$ 1]. A Newman-Keuls test showed that both red and green presamples significantly lowered the accuracy with which short temporal samples were matched relative to the nopresample condition. However, there was no significant difference in the amount of disruption produced by red as opposed to green presamples.

Finally, in the lower right graph of Figure 5 it can be seen that temporal presamples had a slight, but statistically significant, effect on the accuracy with which color target samples were matched $[F(2,28)=4.61, p<.05]$. There was no effect of target sample $[F(1,14)=1.18]$ or presample duration $\times$ target sample interaction $[F(2,28)<1]$. The group factor was not significant, and it did not interact with any other factor.

\section{Discussion}

Given the absence of a significant choose-short effect in Experiment 1 and Phase 1 of Experiment 2, it is surprising that temporal summation effects were obtained whenever temporal target samples were preceded by temporal presamples or color presamples during interference tests. Although temporal summation effects with temporal presamples and temporal target samples have been reported by Spetch and Sinha (1989) and Wilkie and Willson (1990), this is the first study to show that temporal sum- 
mation effects also occur with color presamples. Recent evidence suggests that interference tests with temporal target samples produces evidence of summation regardless of the nature of the memory code for temporal samples. Spetch and Grant (1991) have reported temporal summation effects with a successive MTS procedure even though delay tests failed to reveal a choose-short effect. They suggested that the temporal summation effect is due to a failure of the accumulator to reset to zero prior to the onset of the second sample, rather than being due to a specific type of code's (i.e., retrospective and analogical) being maintained in working memory. Consequently, the second sample is perceived as being longer than a sample of equal duration presented when the accumulator has a count of zero. Kraemer and Roper (1992) have made similar assumptions in showing how a prospective coding model can account for temporal summation effects. The present data on temporal summation is consistent with the results accumulating from other laboratories, that summation effects are obtained even when prior delay tests fail to produce choose-short effects. Most importantly, these summation effects even occur when the relevant dimension of the presample is color rather than duration.

The slight disruption in the matching of color samples when they were preceded by temporal samples of either a short or a long duration was probably a consequence of the illumination associated with temporal samples affecting the encoding of color samples. Support for this interpretation comes from a study by Santi (1984), which demonstrated that ITI houselight illumination disrupted color matching accuracy more when it occurred at the end of the ITI than when it occurred at the beginning of the ITI.

\section{GENERAL DISCUSSION}

The memory code for temporal and nontemporal samples associated with the same comparison choices in an MTO DMTS procedure was examined. In Experiments 1 and 2, the retention functions for temporal and visual samples were compared. As in the Kraemer and Roberts (1984) study, in which the retention of visual stimuli was compared with that of auditory stimuli, it was argued that if information for the different sample dimensions was being encoded differently, then qualitative and/or quantitative differences in the retention of this information would be observed. Presumably, a systematic bias to choose short should be observed following temporal samples, but no systematic response bias should be observed following color samples. Contrary to this prediction, a significant choose-short effect was not observed, and temporal and color samples mapped onto the same correct comparison stimulus had similar retention functions. These data provide indirect evidence of some similarity in the memory codes for temporal and visual information. Certainly, it seems unlikely that the temporal samples were being coded in a strictly analogical fashion.

The nature of the memory code for temporal samples reflected in the present set of findings is open to numer- ous interpretations. One obvious candidate is common prospective coding of a particular comparison stimulus to respond to (Grant, 1982; Santi \& Roberts, 1985). However, Urcuioli et al. (1989) have suggested that the common code does not preserve stimulus information about the comparisons although it is derived from the samples' common comparison associates (e.g., sample A and sample B). Thus, the common code may be an abstract intermediate code that does not contain any specific information about the physical properties of the sample or the comparison stimulus. On the other hand, it is quite plausible that the common code might be isomorphic with one of the two sample dimensions. For example, since hues are so highly discriminable for pigeons, the common code for line samples in Urcuioli et al. and for temporal samples in the present study could have been in a form isomorphic with the hue samples (e.g., 2 sec coded as "red" and 8 sec coded as "green"). Zentall, Steirn, Sherburne, and Urcuioli (1991) have recently presented evidence that when a hue and a line are associated with a common event, both may be represented in memory as the hue. Additional research is needed to identify the precise nature of the common code induced by the MTO procedure.

The intratrial proactive interference procedure used in Experiment 3 does not appear to be sensitive to the nature of code maintained in working memory. Along with the results of Spetch and Grant (1991), it appears that the temporal summation effect arises from nonmemorial properties of the timing system and is independent of the memory code being used. This conclusion is particularly compelling given the present findings that show evidence of temporal summation even when temporal target samples are preceded by color presamples. Although the interference test results do not appear to be diagnostic, the important point is that temporal samples are not retrospectively and analogically coded in the current MTO procedure.

Two important contributions have been made with the present research. In contrast to previous evidence that temporal information is coded in a retrospective and analogical fashion (Spetch \& Sinha, 1989; Wilkie \& Willson, 1990), the present research has identified an additional condition under which temporal information does not appear to be coded in this way. There are now three experimental procedures in which temporal samples are not retrospectively and analogically coded: the present MTO mapping conditions, successive MTS (Grant \& Spetch, 1991), and choice MTS in which temporal samples are associated with differential outcomes of food and no food (Ducharme \& Santi, 1993). In addition, the present interference test results indicate that temporal summation effects are observed even when a choose-short effect has not been observed and most importantly even when the temporal target samples are preceded by color presamples. These findings provide additional support for Spetch and Grant (1991) and Kraemer and Roper's (1992) contention that the memory code for temporal samples cannot be identified on the basis of the occurrence of a 
temporal summation effect in intratrial proactive interference tests.

\section{REFERENCES}

Bowers, R. L., \& Richards, R. W. (1990). Pigeons' short-term memory for temporal and visual stimuli in delayed matching-to-sample. Animal Learning \& Behavior, 18, 23-28.

Cohen, J. S., Galgan, R., \& Fuerst, D. (1986). Retrospective and prospective short-term memory in delayed response tasks in rats. Animal Learning \& Behavior, 14, 38-50.

Cohen, J. S., Grassi, J., \& Dowson, P. (1988). The role of withintrial location of the retention interval in rats' delayed conditional discrimination performance. Animal Learning \& Behavior, 16, 40-46.

Ducharme, M. J., \& Santi, A. (1993). Alterations in the memory code for temporal events induced by differential outcome expectancies in pigeons. Animal Learning \& Behavior, 21, 73-81.

Grant, D. S. (1982). Prospective vs retrospective coding of samples of stimuli, responses, and reinforcers in delayed matching with pigeons. Learning \& Motivation, 13, 265-280.

Grant, D. S. (1991). Symmetrical and asymmetrical coding of food and no-food samples in delayed matching in pigeons. Journal of Experimental Psychology: Animal Behavior Processes, 17, 186-193.

Grant, D. S., \& MacDonald, S. E. (1990). An evaluation of the role of dual coding in mediating the effect of incorrectly cuing the comparison dimension in delayed matching in pigeons. Animal Learning \& Behavior, 18, 151-156.

Grant, D. S., \& SPETCh, M. L. (1991). Pigeons' memory for event duration: Differences between choice and successive matching tasks. Learning \& Motivation, 22, 180-199.

HoNIG, W. K., DoDD, P. W. D. (1983). Delayed discriminations in the pigeon: The role of within-trial location of conditional cues. Animal Learning \& Behavior, 11, 1-9.

HoNIG, W. K., \& DoDD, P. W. D. (1986). Anticipation and intention in working memory. In D. F. Kendrick, M. E. Rilling, \& M. R. Denny (Eds.), Theories of animal memory (pp. 77-100). Hillsdale, NJ: Erlbaum.

HoniG, W. K., Thompson, R. K. R. (1982). Retrospective and prospective processing in animal working memory. In G. H. Bower (Ed.), The psychology of learning and motivation: Advances in research and theory (Vol. 16, pp. 239-283). New York: Academic Press.

Honig, W. K., \& Wasserman, E. A. (1981). Performance of pigeons on delayed simple and conditional discriminations under equivalent training procedures. Learning \& Motivation, 12, 149-170.

Kraemer, P. J., Mazmanian, D. S., \& Roberts, W. A. (1985). The choose-short effect in pigeon memory for stimulus duration: Subjective shortening versus coding models. Animal Learning \& Behavior, 13, 349-354

Kraemer, P. J., Roberts, W. A. (1984). Short-term memory for visual and auditory stimuli in pigeons. Animal Learning \& Behavior, 12, 275-284.

Kraemer, P. J., \& Roper, K. L. (1992). Matching-to-sample performance by pigeons trained with visual-duration compound samples. Animal Learning \& Behavior, 20, 33-40.

PARKer, B. K., \& Glover, R. L. (1987). Event duration memory: The effects of delay-interval illumination and instructional cuing. Animal Learning \& Behavior, 15, 241-248.

Ponteconvo, M. J. (1985). Memory for a stimulus versus anticipation of a response: Contrasting effects of proactive interference in two delayed comparison tasks. Animal Leaming \& Behavior, 13, 355-364.

RoITBLAT, H. L. (1980). Codes and coding processes in pigeon shortterm memory. Animal Learning \& Behavior, 8, 341-351.

RILEY, D. A., COOK, R. G., \& LAMB, M. R. (1981). A classification and analysis of short-term retention codes in pigeons. In G. H. Bower (Ed.), The psychology of learning and motivation: Advances in research and theory (Vol. 15, pp. 51-79). New York: Academic Press.
SANTI, A. (1984). The trial spacing effect in delayed matching-to-sample by pigeons is dependent upon the illumination condition during the intertrial interval. Canadian Journal of Psychology, 38, 154-165.

SantI, A., Musgrave, S., \& Bradford, S. A. (1988). Utilization of cues signalling different test stimulus dimensions in delayed matching by pigeons. Learning \& Motivation, 19, 87-98.

Santi, A., \& Roberts, W. A. (1985). Prospective representation: The effects of varied mapping of sample stimuli to comparison stimuli and differential trial outcomes on pigeons' working memory. Animal Leaming \& Behavior, 13, 103-108.

SPETCH, M. L. (1987). Systematic errors in pigeons' memory for event duration: Interaction between training and test delay. Animal Learning \& Behavior, 15, 1-5.

SPETCH, M. L., \& GRANT, D. S. (1991, June). Memory for time in successive vs. choice tasks. Paper presented at the meeting of the Canadian Society for Brain, Behaviour, and Cognitive Science, Calgary, Alberta.

SPETCH, M. L., \& RuSaK, B. (1989). Pigeons' memory for event duration: Intertrial interval and delay effects. Animal Learning \& Behavior, 17, 147-156.

SpeTCH, M. L., \& Sinha, S. S. (1989). Proactive effects in pigeons' memory for event durations: Evidence for analogical retention. Joumal of Experimental Psychology: Animal Behavior Processes, 15, 347-357.

SPETCH, M. L., \& WILKIE, D. M. (1982). A systematic bias in pigeons' memory for food and light durations. Behaviour Analysis Letters, 2, 267-274.

SPETCH, M. L., \& WILkIE, D. M. (1983). Subjective shortening: A model of pigeons' memory for event duration. Joumal of Experimental Psychology: Animal Behavior Processes, 9, 14-30.

Stonebraker, T. B., \& Rilling, M. (1984). Retrospective versus prospective processes in delayed matching to sample. Bulletin of the Psychonomic Society, 22, 372-375.

UrCuioli, P. J., \& Zentall, T. R. (1986). Retrospective coding in pigeons' delayed matching-to-sample. Journal of Experimental Psychology: Animal Behavior Processes, 12, 69-77.

Urcuioli, P. J., \& ZENTALl, T. R. (1990). On the role of trial outcomes in delayed discriminations. Animal Learning \& Behavior, 18, 141-150.

Urcuioli, P. J., Zentall, T. R., Jackson-Smith, P., \& Steirn, J. N. (1989). Evidence for common coding in many-to-one matching: Retention, intertrial interference, and transfer. Journal of Experimental Psychology: Animal Behavior Processes, 15, 264-273.

Wasserman, E. A., (1986). Prospection and retrospection as processes of animal short-term memory. In D. F. Kendrick, M. E. Rilling, \& M. R. Denny (Eds.), Theories of animal memory (pp. 53-75). Hillsdale, NJ: Erlbaum.

Weisman, R. G., Bruce, R., \& Beninger, R. J. (1987). Simple and conditional discrimination in rats: The effects of delays and scopolamine. Learning \& Motivation, 18, 274-287.

WILKIE, D. M., \& WILLSON, R. J. (1990). Discriminal distance analysis supports the hypothesis that pigeons retrospectively encode event duration. Animal Learning \& Behavior, 18, 124-132.

Zentall, T. R., JAGIELo, J. A., Jackson-Smith, P., \& URCuiou, P. J, (1987). Memory codes in pigeon short-term memory: Effects of varying the number of sample and comparison stimuli. Learning \& Motivation, 18, 21-33.

Zentall, T. R., Steirn, J. N., Sherburne, L. M., \&rculoli, P. J. (1991). Common coding in pigeons assessed through partial versus total reversals of many-to-one conditional and simple discriminations. Journal of Experimental Psychology: Animal Behavior Processes, 17, 194-201.

Zentall, T. R., Urculol, P. J., JaGielo, J. A., \& Jackson-Smith, P. (1989). Interaction of sample dimension and sample-comparison mapping on pigeons' performance of delayed conditional discriminations. Animal Learning \& Behavior, 17, 172-178.

(Manuscript received July 10, 1992; revision accepted for publication October 16, 1992.) 